Málaga).Acta Bot. Malacitana 18: 199-221. APARICIO, A., SILVESTRE, S. -1987-Flora del Parque Natural de la Sierra de Grazalema. Junta de Andalucía.

BLANCA, G., B. CABEZUDO, J.E. HERNÁNDEZBERMEJO, C.M. HERRERA, J. MUÑOZ y B. VALDÉS -2000-Libro Rojo de la Flora Silvestre Amenazada de Andalucía. Especies Vulnerables. Sevilla.
BECERRA, M., J.A. GARCÍA ROJAS, A.V. PÉREZ LATORRE, B. CABEZUDO y T. NAVARRO 2001- Aportaciones a la flora de Andalucía. Acta Bot. Malcitana 26: 284-286.

Aceptado para su publicación en octubre de 2002

Dirección del autor. Dpto. Biología Vegetal. Facultad de Ciencias. Universidad de Málaga Campus de Teatinos. 29071, Málaga.

\title{
86. NUEVAS CITAS DE MONOCOTILEDÓNEAS PARA LA PROVINCIA DE GRANADA (SUR DE ESPAÑA).
}

\author{
Maria Teresa VIZOSO, Laura BAENA y Francisco Bruno NAVARRO
}

New floristic records of Monocotyledons for Granada province (S Spain).

Palabras clave. Corología, Monocotiledóneas, Granada.

Key words. Corology, Monocotyledons, Granada, Spain.

A pesar de las numerosas campañas de herborización realizadas en la provincia de Granada, las monocotiledóneas y más concretamente algunos geófitos y terófitos, a veces han pasado desapercibidas dada su estacionalidad y efímera floración. La continua revisión de los fondos del Herbario de la Universidad de Granada (GDA), así como la elaboración de sus bases de datos, nos han permitido detectar testimonios no publicados y otros que corroboran la existencia de especies consideradas raras en nuestra provincia. Entre ellos, se citan por primera vez para Granada Iris subbiflora, Narcissus bugei, Stenberngia colchiciflora y Ventenata dubia. Para la comprobación de las citas se han consultado los Archivos de Flora Ibérica (Corología Ibérica, I-IV) [Velayos et al. (1991-1992) y Velayos \& Castilla (1993)], así como revisiones recientes y diversas notas corológicas que se especifican en el texto.

\section{Colchicum lusitanum Brot.}

GRANADA: Loja, Sierra de Loja, Charco Negro, 30SUG9508, prados pedregosos, 19-X-1988, M. T. Vizoso \& M. Zea, GDA 44163. Ibídem, Paso de la Burra, 30SUG9512, $990 \mathrm{~m}$, matorral serial nitrificado, sustratos calizos, 29-II-1989, M. V. García, J. García \& M. J. Salinas, GDA 44162. Güéjar Sierra, La Trinchera, 30SVG6013, prados pedregosos, 19-X-1988, M. T. Vizoso \& M. Zea, 
GDA 44179. Sierra de Cázulas, sustrato calcáreo, 2-X-1994, M. Ruíz Rejón, GDAC 38539. Sierra Elvira, $850 \mathrm{~m}$, sustrato calcáreo, 5-IX-1996, G. Blanca, M. J. Marínez \& M. J. Salinas, GDAC 40227. Montefrío, Peña de los Gitanos, 30SVG1533, $960 \mathrm{~m}$, claros de encinar sobre sustrato calizo, 8-X2000, C. Morales, GDA 43867.

Este geófito de distribución Ibérica, del norte de África y centro de Italia, se encuentra poco referenciado para la mitad septentrional de la Península, de donde se conoce citado para Lugo, Orense y Salamanca; sin embargo en Andalucía se presenta en casi todas las provincias, siendo más abundante en las occidentales. Fernández et al. (1991) to indicaron para Granada. Con los restimonios aportados queda confirmada su presencia en las sierras calizas granadinas.

\section{Iris subbiflora Brot.}

GRANADA: Loja, Sierra de Loja, Cerro de la Picharra, 30SUG9712, $1000 \mathrm{~m}$, prados pedregosos, 14-V-1989, M. V. Garcia \& M. J. Salinas, GDA 44176. Ibídem, en rocas calizas, 5-IV-1989, M. V. García, J. Peñas \& M. J. Salinas, GDA 44177.

Primeras citas para Granada de esta especie endémica del SW español y el centro de Portugal. En Andalucía se ha citado en diversas sierras calizas de Córdoba, Málaga, Sevilla, Jaén y Almería. En la Sierra de Loja es escaso, pero localmente frecuente, y convive con Iris planifolia (Miller) Fiori et Paol., muy común y con floración más temprana, de diciembre a marzo.

Narcissus bugei (Fernández Casas) Fernández Casas GRANADA: Loja, Sierra de Loja, próximo Charco de Alcantar, 30SUG9408, $1250 \mathrm{~m}$, cascajares calizos, 12-III-1989, M. V. García \& M. J. Salinas, GDA 44173. Ibídem, Charco Negro, 30SUG9508, 13-V-1989, M. V. Garcia, GDA 44174. Ibídem, Cerro de la Corona, 30SUG91, 850-950 m, sustratos calizos, en umbrías de encinar en recuperación, 22II-2001, L. García Cardenete \& P. Lanzas Quintana, GDA 45789.

Endemismo andaluz de las Subbéticas cordobesas, de la Sierra de Jabalcuz (Jaén; Blanca, com. pers.) y de la Sierra de las Nieves (Serranía de Ronda, Málaga), que amplía su área de distribución a la Sierra Gorda de Loja y cerros próximos. A pesar de que estas dos nuevas poblaciones tienen características ecológicas algo diferentes entre sí (las poblaciones de la Sierra Gorda se desarrollan sobre prados pedregosos muy expuestos y sin apenas ninguna cubierta vegetal, mientras que la población del Cerro de la Corona, mucho más extensa y con mayor densidad, se encuentra en lugares umbrios bajo un encinar en franca recuperación), ambas cumplen los requerimientos ecológicos básicos de esta especie, suelos estacionalmente algo húmedos sobre sustratos pedregosos calizos y altitudes alrededor de los $1000 \mathrm{~m}$. o superiores.

Está considerada especie en peligro de extinción (Hernández-Bermejo et al., 1999), ya que se trata de poblaciones muy localizadas y amenazadas por la presión humana debida al turismo verde, expediciones de recolectores e ingestas de jabalíes. Dado que las nuevas poblaciones no se encuentran localizadas en ningún espacio natural protegido, convendría evaluarlas y, en caso de que fuera conveniente, incluirlas en los planes de recuperación de esta especie.

\section{Orchis champagneuxii Barnéoud}

GRANADA: La Bernardilla, 30SVF7551, prados en claros de matorral, 24-III-1989, M. T. Vizoso \& J. C. de la Rosa, GDA 44019.

Frecuente en el norte y centro peninsular y escasa o rara en Andalucía, aunque está bastante citada en el norte de la provincia de Jaén. En Granada, sólo se conoce la cita de Vélez de Benaudalla (Molero Mesa et al., 1981), siendo ésta la segunda cita provincial.

\section{Orchis ustulata $\mathrm{L}$.}

GRANADA: Alhama de Granada, Cortijo de Lapachares, la Alcaicería, alrededores del Arroyo de la Madre, 30SVF0889, $1075 \mathrm{~m}$, claros del matorral, sobre sustrato dolomítico, 2-V-2000, L. Baena \& L. de Manuel Jerez, GDA 45547.

En la Península Ibérica se distribuye por el C y NE, alcanzando hacia el Sur las provincias de Córdoba y Granada. Molero \& Aroza (1984), la citaron en la Sierra de Harana (Granada), por lo que el testimonio aportado constituye la segunda cita provincial.

\section{Romulea columnae Sebast. \& Mauri}

GRANADA: Armilla (Base Aérea), 30SVG4210, prados secos, 5-III-1989, M. T. Vizoso \& J. C. de la Rosa, GDA 44167. La Herradura, Cerro Gordo, prados húmedos nitrificados, 22-I- 
1998, C. Morales, C. Quesada, L. Baena \& J. E. Linares, GDA 42419.

Se trata de una especie rara en Andalucía, conocida de Cádiz, Sevilla y Almería. En Granada solo se había citado en Órgiva (Molero Mesa \& Pérez Raya, 1987). Concretamente la cita de Cerro Gordo no hace más que corroborar su notable distribución mediterránea y su preferencia por tierras bajas con cierta termicidad o influencia costera, como ponen de manifiesto las citas a lo largo del mediterráneo, desde Gerona hasta Almería o en las costas atlánticas y cantábricas.

Stenberngia colchiciflora Waldst. \& Kit.

GRANADA: Güéjar Sierra, La Trinchera, 30SVG6013, prados pedregosos, 19-X-1988, M. T. Vizoso \& M. Zea, GDA 44179. Dílar, próximo a Ermita Vieja, 30SVF5000, $1400 \mathrm{~m}$, zona húmeda bajo pinar, 29-XI-1988, A. Rupérez Rupérez \& $R$. Salas Martin, GDA 44172.

Especie bastante citada para el centro de España y la cuenca del Duero, pero no tan frecuente en Andalucía de donde se conoce citada en la mitad occidental (Cádiz, Huelva, Málaga y Sevilla), siendo estas las primeras citas para la provincia de Granada.

Ventenata dubia (Leers) Cosson

GRANADA: Sierra de Castril, Suerte Somera, 30SWH2702, $1800 \mathrm{~m}$, prados húmedos moderadamente nitrificados, 14-VII-1992, C. Morales \& C. Passera, GDAC 37101.

Terófito poco citado en la Península, conocido fundamentalmente del piso supramediterráneo de las montañas del centro (Ávila, Guadalajara, Segovia y Soria). En Andalucía solo la citó Colmeiro (1889), en una localidad jiennense próxima a Granada. Con este testimonio se confirma la presencia de este taxón en la provincia granadina.

\section{BIBLIOGRAFÍA}

COLMEIRO, M. - 1889- Enumeración y revisión de las plantas de la Península Hispano-lusitana e Islas Baleares. Tomo V. Madrid.

FERNÁNDEZ, C., M. C. CRUZ, M. LÓPEZ PULIDO, C. AMESCUA y D. CASADO - 1991Flora de Andalucía, Catálogo bibliográfico de las plantas vasculares. Facultad de Ciencias Experimentales de Jaén.
HERNÁNDEZ-BERMEJO, E., M. A. GARCÍA, P CONTRERAS y M. CLEMENTE -1999Narcissus bugei. In Blanca, G. et al. Libro rojo de la flora silvestre amenazada de Andalucía. Tomo I: Especies en peligro de extinción. Pp.: 87-190. Consejería de Medio Ambiente. Junta de Andalucía.

MOLERO MESA, J. y P. AROZA -1984- Dos orquídeas de Granada. Anales Jard. Bot. Madrid, 41(1): 205.

MOLERO MESA, J. y F. PÉREZ RAYA - 1987- La flora de Sierra Nevada. Avance sobre el catálogo florístico nevadense. Serv. Publ. Univ. Granada.

MOLERO MESA, J., F. A. PÉREZ RAYA y J. M. MARTÍNEZ PARRAS - 1981-Relación de las Orchidaceae de la provincia de Granada. Anales Jard. Bot. Madrid 37(2): 645-659.

VELAYOS, M., F. CASTILLA y R. GAMARRA (eds.) -1991- Archivos de Flora Ibérica. Núm. 2. (Corología Ibérica, I). Real Jardín BotánicoICYT. CSIC. Madrid.

VELAYOS, M., F. CASTILLA y R. GAMARRA (eds.) -1991-Archivos de Flora Ibérica. Núm. 3. (Corología Ibérica, II). Real Jardín BotánicoICYT. CSIC. Madrid.

VELAYOS, M., F. CASTILLA y R. GAMARRA (eds.) -1992-Archivos de Flora Ibérica. Núm. 5. (Corología Ibérica, III). Real Jardín BotánicoICYT. CSIC. Madrid.

VELAYOS, M. y F. CASTILLA (eds.) -1993Archivos de Flora Ibérica. Núm. 6. (Corología Ibérica, IV). Real Jardín Botánico- ICYT. CSIC. Madrid.

Aceptado para su publicación en septiembre de 2002

Dirección de los autores: M. T. VIZOSO y L. BAENA: Herbario (Dpto. Botánica). Fac. Ciencias. Universidad de Granada, Fuentenueva, s/nº, 18001 Granada. e-mail: herbario@ugr.es. F. B. NAVARRO: Centro de Investigación y Formación Agraria, Camino de Purchil s/n ${ }^{\circ}, 18080$ Granada. email: cifafore@teleline.es. 